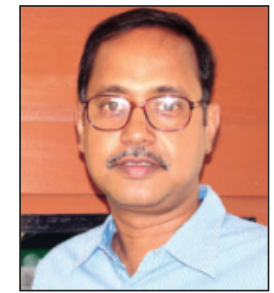

JOURNAL OF MINES, METALS \& FUELS www.jmmf.info f

INDUSTRY DIRECTIONS

Jayanta Bhattacharya

Hony. Chief Editor

\title{
Why ground penetrating radar (GPR) should be allowed for testing in the mines
}

Of these various geophysical sensing techniques, GPR emerges as strong candidate based on its fundamental operating characteristics, subsurface imaging capability, spatial and time resolution, physical size, electromagnetic safety, and functional range. GPR has also been used for a wide variety of applications such as archaeology, civil engineering, hydrology and defence.

GPR utilises the same fundamental time-of-flight measurement principle as in conventional through-air radar, however the radar antennas are placed such that the electromagnetic energy is radiated directly into the ground. When this wave energy interacts with an interface boundary, some of the wave energy may be reflected or scattered. By measuring the (two-way) time period between the transmitted and received waves, and knowing the wave propagation velocity, the distance between the radar and the object can be computed. This basic radar distance measurement principle is shown in the below.

(a) Idealised radar returns corresponding to reflections at the boundaries of horizontally-layered strata geology at spatial positions at $\mathrm{d} 1, \mathrm{~d} 2$ and $\mathrm{d} 3$.

(b) The specific depth to the target subsurface interface can be determined using GPR by finding $d$, where $d$ is the depth to underlying interface, $\mathrm{v}$ is the wave propagation velocity through the transmission medium, $t$ is the twoway travel time of the received electromagnetic signal, and $\mathrm{x}$ is the distance between the transmit and receive antennas.

GPR is gaining acceptance as standard practice for reconnaissance alluvial exploration. The resistive soils of most alluvial enviromnents yield impressive penetration depths and profile resolution. Faster and more representative of geology than test pits and boreholes alone, GPR may be utilised to model the three-dimensional extent of gold and diamond-bearing fluvial sequences. A variety of sediment

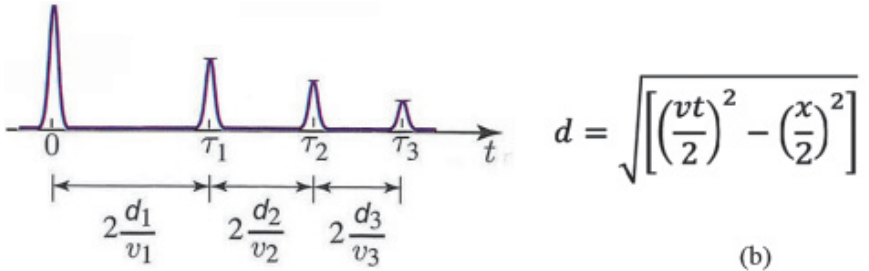

(a)

structures including channel fills; cross-beds and longitudinal bar foresets are commonly identified. In these environments, penetration with the radar is generally excellent and frequently exceeds $40 \mathrm{~m}$. These data may then be used in a mining package for the $3 \mathrm{D}$ reconstruction of the paleochannel system. The data can be used to delineate the extent of the paystreak, to estimate resource volume and to select strategically placed drilling and exploration targets.Radar images have shown that the boundaries of the layering are surprisingly variable between boreholes. These abrupt changes in horizon elevations are generally undetectable by drilling at any economic spacing. By using GPR to accurately map the volume of the deposit, coupled with sparser drilling to confirm the layer identification and grade, a geoscientifically sound resource estimate may be made quickly and economically.In mining, GPRs are being actively used in various mining horizon sensing.

One important thing about adopting such useful technology is to acquaint the people in the mines of the modern technology, particularly automation and digital communication. Such practices would help them adapt to new technologies, to be not fearful and hesitant. Mines should consider gradual introduction of these technologies before adopting complete automation.

Source :https://ieeexplore.ieee.org/ search/ searchresult.jsp? newsearch= true\&queryText $=$ Ground $\% 20$ Penetrating $\% 20$ Radar 